\title{
Novel 4D Whole Body Scanning Solution and its Medical Application
}

\author{
Paweł LIBERADZKI, Łukasz MARKIEWICZ*, Marcin WITKOWSKI, Robert SITNIK \\ Warsaw University of Technology, Mechatronics Faculty, Warsaw, Poland
}

DOI: $10.15221 / 18.047$ http://dx.doi.org/10.15221/18.047

\begin{abstract}
One of the drawbacks of the traditional 3D whole body scanning is that it is capable of capturing only static models. In most of the cases it is impossible to properly analyze the way people move as they are not able to freeze their movement for a certain amount of time. In order to add a 4th dimension (time) to measurements, a system have to be built using stable ultra-fast 3D scanners. The presented solution meets industrial requirements for $4 \mathrm{D}$ measurements of dynamic objects. It is capable of acquiring up to $120 \mathrm{~Hz}$ sequences of high precision point clouds along with an information about its lightness and normal vectors. A spatial resolution of $1 \mathrm{~mm}$ is obtained with an inaccuracy below $0.5 \mathrm{~mm}$. It was originally designed for a 4D human body shape measurement to support medical rehabilitation monitoring, however it is not restricted to this application.
\end{abstract}

The system is composed of four directional measurement columns [1]. Sufficient body surface coverage is possible thanks to an even distribution of modules, each consisting of 1 projector and 2 detectors - on the upper and lower part of the head. Their working principle is based on a structured light projection, specifically a single frame pattern approach which enabled achievement of the declared frequency. For this particular case a problem of synchronization (highly erroneous overlapping of the projected patterns) was solved. A sine modulated patterns are colored and distinguished using a spectral separation via color filters. Information about fringe numbers is encoded using an additional transverse modulation of the patterns. Retrieval of a single multidirectional output cloud is done using a set of dedicated algorithms, including phase unwrapping on a single image per detector, scaling into $\mathrm{XYZ}$ coordinates and common calibration.

The high precision 4D data is very heavy. A raw 1 minute of $120 \mathrm{~Hz}$ scan requires around $360 \mathrm{~GB}$ of a disk space. In order to handle such data, the specialized software called FRAMES (Framework and Robust Algorithms for Models of Extreme Size) was developed. It has built-in 4D RAM (Random Access Memory) manager which enables efficient visualization, advanced multithread processing and analysis of such data.

The presented 4D scanning solution was tested in a real-life environment. The possibility of performing $3 \mathrm{D}$ body scanning in time enabled the rehabilitation progress monitoring after leg amputation.

Keywords: 4D body scanning, 4D data processing, structured light, multidirectional system, medical monitoring

\section{Introduction}

A three-dimensional (3D) scanning enables an acquisition and a precise measurement of a shape and appearance of a physical object. It is commonly used in a wide variety of fields of science and technology. Modern fast and accurate 3D scanners are used, inter alia, in cultural heritage for documentation [2], in law enforcement for crime scene reconstruction [3], and for the whole human body measurements in medicine [4,5] and anthropometry [6]. However, the human body is always in a motion, thus it is a challenge to properly capture and analyze it using $3 \mathrm{D}$ scanners. A four-dimensional (4D) information, that is a 3D data over an extended period of time, was firstly introduced within systems capturing only the location of fiducial markers attached to the human skin. These systems have been successfully used in many motion-capture applications [7, 8]. Then, along with an improvement of the optoelectronic devices and an increase in computing power of computers, 4D scanners started to be able to capture the whole surface of the human body in motion. The major approaches usually utilize one of the following techniques:

- Time-of-Flight (ToF) [9] - based on a return time of a light impulse,

- Structure from Motion (SfM) [10] - based on an analysis of images of the same object that are taken from different directions and at the same time,

- Laser Triangulation (LT) [11] - based on an analysis of laser stripes projected onto the object surface,

- Structured Light (SL) [12] - based on an analysis of a deformation of a certain raster projected onto the object surface. 
Some of the leading 3D scanning companies utilized these techniques and released their commercial 4D systems which were then used in numerous research (e.g. Diers International GmbH [13, 14], $3 \mathrm{dMD}$ LLC [15, 16], and Microsoft Corporation [17]). However, there is still much room for improvements, because these systems have various limitations, i.e. low acquisition frequency and precision or high equipment cost. In this paper, authors present a 4DBODY scanner - a novel 4D whole body scanning system based on the SL technique. The proposed system is characterized by a high measurement spatial resolution of $1.0 \mathrm{~mm}$ with an accuracy of $0.5 \mathrm{~mm}$ and an acquisition frequency of up to $120 \mathrm{~Hz}$. Moreover, its construction is simple and does not require dozens of expensive devices. Finally, it is also equipped with an advanced $3 D$ and $4 D$ data processing and visualization software called FRAMES (Framework and Robust Algorithms for Models of Extreme Size) which enables convenient work and analysis of data-intensive scans.

\section{Materials and methods}

\subsection{System architecture}

The proposed 4D measurement system utilizes the SL technique because of its high accuracy and reliability. Moreover, our team's experience in this field is significant which was proved before [18, 19]. The SL based application requires using both detectors and projectors. It was decided to assemble them into Measurement Heads (MHs). To enable reconstruction of the whole human body (including most problematic regions like shoulders or groin), four of the MHs are used, each consisting of two detectors and one projector, as it is depicted in figure 1. As for detectors, Grasshopper 3.0 - fast, industrial cameras produced by FLIR Integrated imaging Solutions Inc. [20] were chosen, because they provide up to $120 \mathrm{HZ}$ of acquisition frequency in an external trigger mode. In case of projector, a custom modified one was prepared based on a Casio XJ-A242 device [21]. Each pair of the MHs (belonging to a front or a rear frame) is equipped with an independent PC-class processing unit that is responsible for a captured data management. A local network, where each of the frame units acts like a server, is responsible for a reciprocal communication between the devices. The operator can control the system using a master processing unit with a dedicated client application.

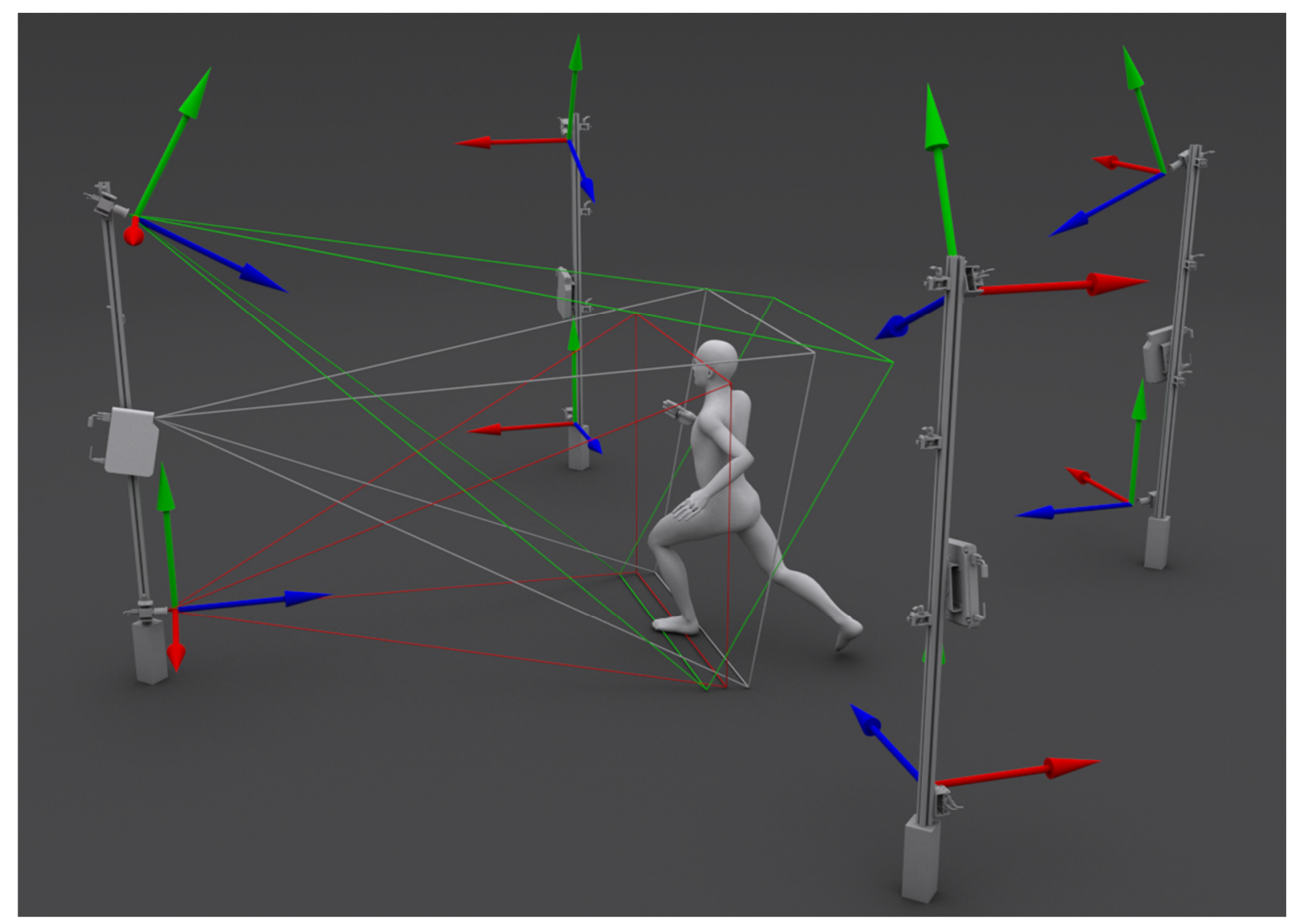

Fig. 1. An architecture of the proposed measurement system. 
In the proposed system, the synchronization between the devices is done using one Atmel AVR microcontroller [22] together with four Terasic FPGAs (Field-Programmable Gate Arrays) [23]. Each FPGA is responsible for a single projector pattern projection management and simultaneous triggering of the $\mathrm{MH}$ detectors. The microcontroller synchronizes multiple FPGAs tasks in order to ensure that all of the frames are captured at the same moment.

In the SL technique, each detector-projector pair within a single module behaves like a triangulation pair. For this reason, it is necessary to ensure that the projected pattern is captured only by the adequate detector. In order to avoid overlapping of the projected patterns, spectral separation was applied. Color filters are used to reduce the range of wavelengths captured by the cameras. The color of the projected patterns is set to blue or red (alternately for neighboring MHs). This way the proper distinction of the measurement frames by each of the detectors is ensured.

\subsection{Reconstruction algorithms}

The developed system working principle is the SL single frame method based on a sine fringe pattern. This approach is an alteration of the method proposed by Sitnik [24]. The modified representation of a distinguished fringe is proposed, as it is presented in figure 2. The distinguished fringe, called further a marker, carries information about a fringe number which is necessary for a proper phase reconstruction. This problem usually does not occur in multiple frame methods, where this information is mostly explicit.
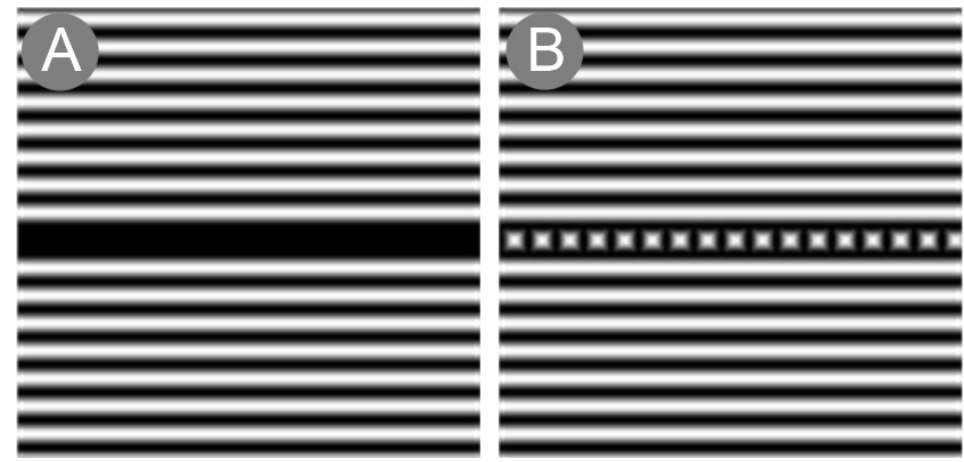

Fig. 2. A comparison of the sine fringe patterns: A) originally introduced by Sitnik [21];

$B)$ modified version proposed by the authors.

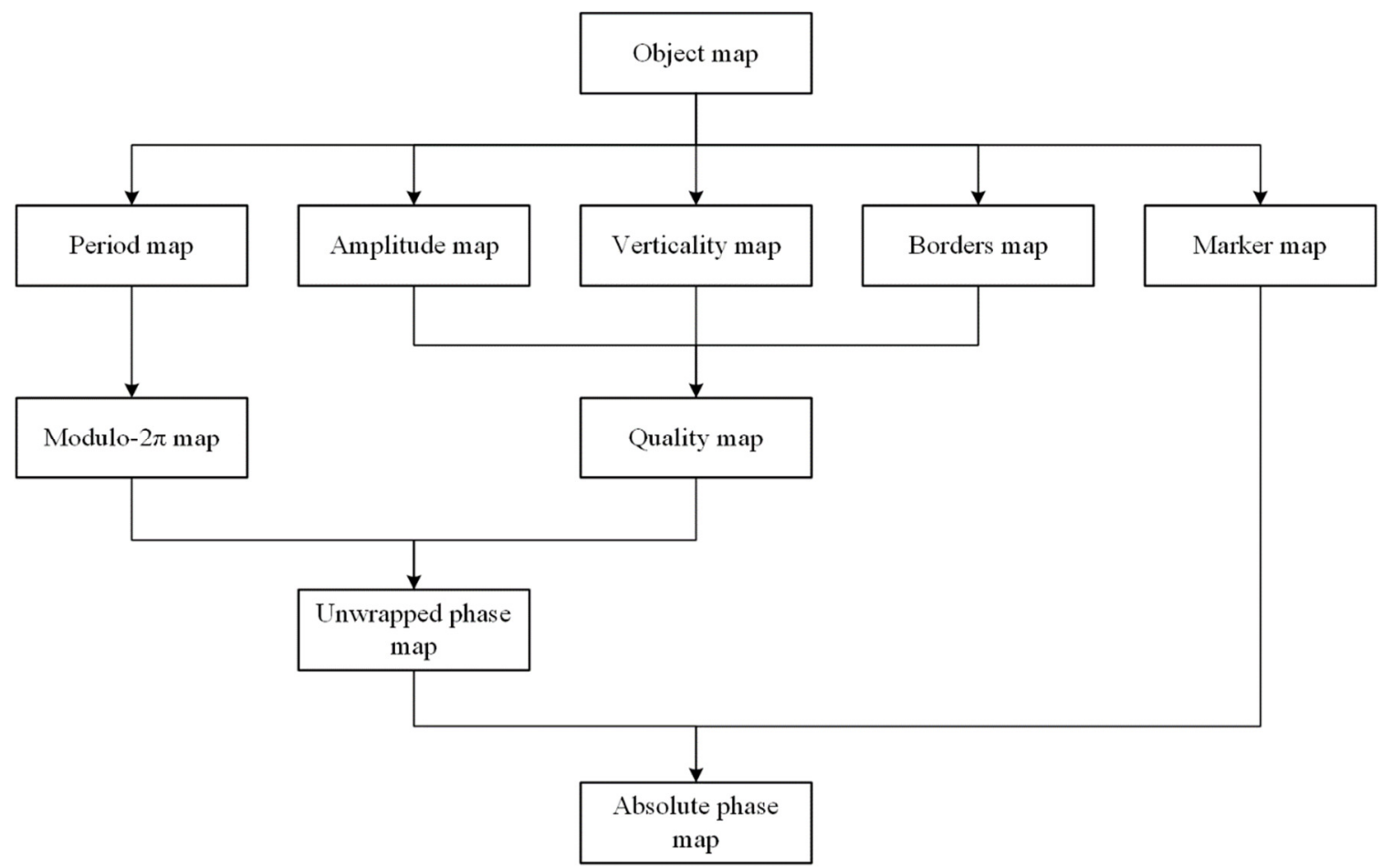

Fig. 3. A processing flowchart for a single frame. 
The data is processed according to the diagram presented in figure 3 . The procedure begins with calculating the ob ject map calculation, such as it is depicted in figure 4B. In this step, the measured area is separated from the background. This way further processing can be restricted only to necessary pixels, significantly reducing computation time. The background separation is performed using an Otsu thresholding [25] and followed by a closing operation.
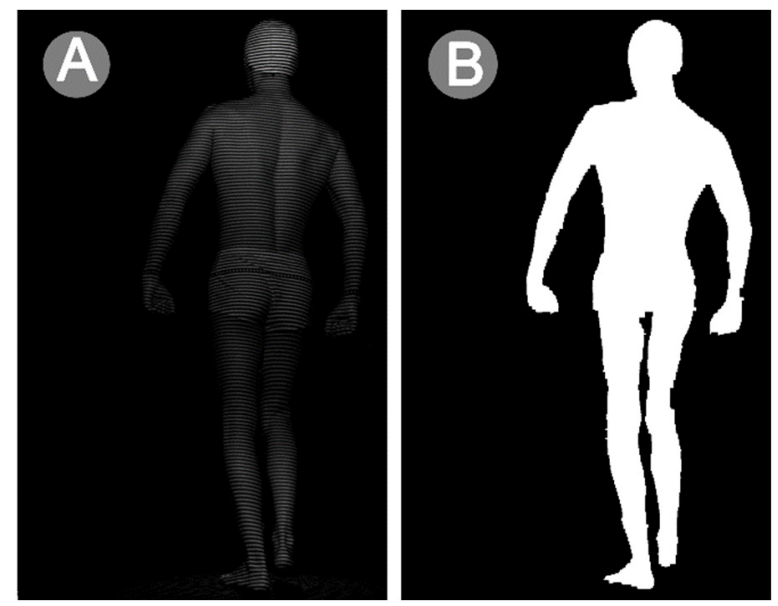

Fig. 4. An example of the background separation: A) Input image; B) ob ject map.

In the next step, Period, Amplitude, Verticality, and Borders maps are prepared. The Period map calculation starts with a local neighborhood median pixel intensity computation. The output is then used to evaluate a local fringe period. The resulting value is an average of distances between subsequent "intersections" of image pixel intensities and the median (see figure 5A). Simultaneously, the Amplitude, Verticality and Borders maps are calculated. The Amplitude map pixel values are equal to the difference between the maximum and minimum intensity in the local neighborhood, as it is depicted in figure 5B. The Verticality map is related to a sine fringe direction stability. The calculation of this map utilizes information of vertical and horizontal intensity gradients. The pixel value in this case is equal to the ratio between the gradient values and is maximal for the horizontal fringe direction, as it is shown in figure 5C. Finally, the Borders map is simply a Gaussian blurred ob ject map, as in figure 5D.
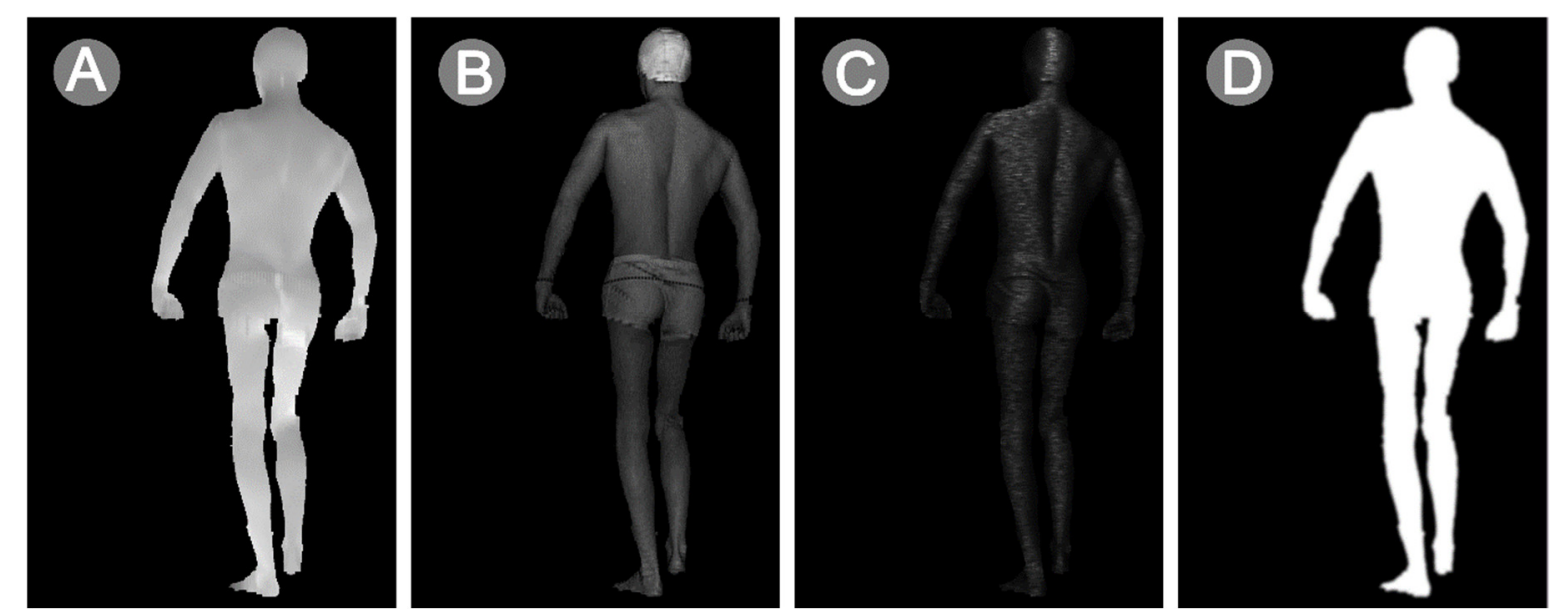

Fig. 5. An example calculation results of the certain maps:

A) Period; B) Amplitude; C) Verticality; D) Borders.

Next, the Modulo-2п map is calculated using the Period map (see figure 6A). In this step, a seven-point SCPS (Spatial Carrier Phase Shifting) method proposed by Larkin is used [26]. It was selected because it is resistant to an inaccurate intensity sampling. Subsequently, the Quality map (see figure 6B), is calculated as an average of the following normalized maps:

- Amplitude - favors areas where the sine fringes have high contrast,

- Verticality - favors areas where the singe fringes orientation is constant,

- Borders - favors areas that are distant to the edges of the object. 

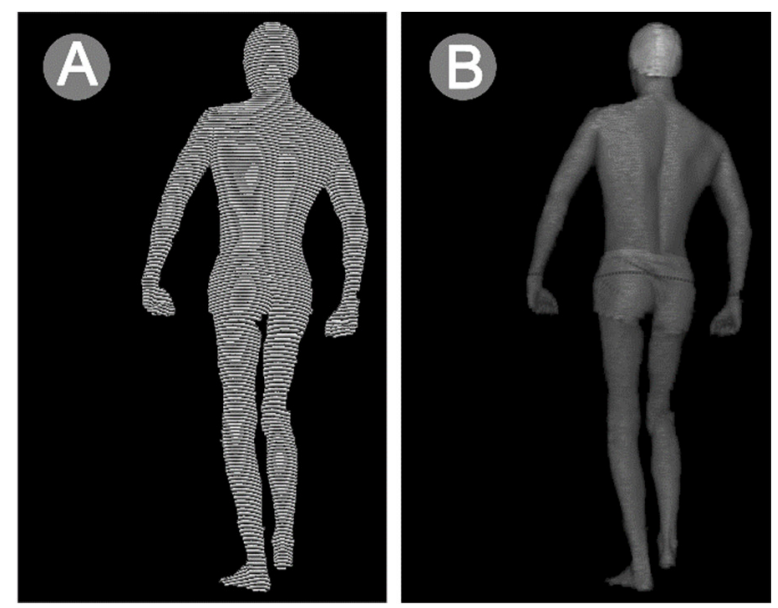

Fig. 6. An example map calculation results: A) Modu lo-2п; B) Qua lity.

Both Modulo-2 $\mathrm{m}$ and Quality maps are used to perform a phase unwrapping. The example of the Unwrapped phase map is presented in figure 7A. Its calculation begins with a random pixel selection from the top $20 \%$ of the highest intensity Quality map pixels. This pixel is then used as an initial point for a Minimum Spanning Tree (MST) algorithm [27]. The MST branch weights are taken directly from the Quality map. As a result, a local phase distribution is generated. However, phase values have to be shifted to obtain a final distribution. For this purpose, information about the marker number is needed. It can be obtained from the Marker map. The marker is localized using 1D FFT (One-dimensional Fast Fourier Transform) [28] filtering in the horizontal direction, as it is shown in figure 7B. Processed pixels are used to calculate the desired phase shift which is afterwards applied to the Unwrapped phase map. This way the Absolute phase map is obtained.
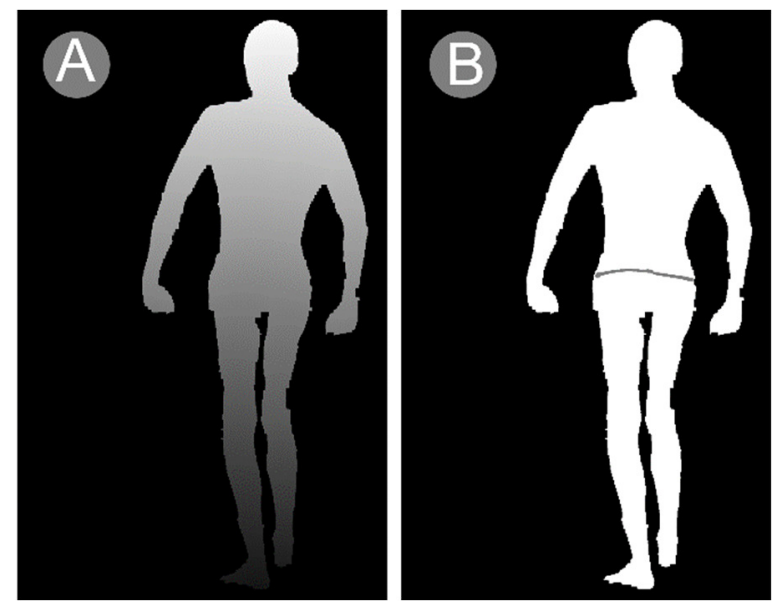

Fig. 7. An example map calculation results: A) Unwrapped phase; B) Marker.

The real world coordinates are calculated using both Absolute phase map and system calibration data. Such output is formed as a point cloud and saved for a further processing.

The proposed scanning solution is capable of acquiring eight single-directional point clouds, one per each camera. These clouds have to be merged into one dense multidirectional cloud based on transformations between the detector and local coordinate systems. This process is called a global system calibration. It begins with a measurement of an arbitrary object, e.g. some calibration artefact or even a human. The received point clouds are then fitted to each other using Iterative Closest Point (ICP) algorithm [29]. This way all of the relative transformations can be calculated. They are stored as a system global calibration and are applied to further measurements.

\subsection{Processing and visualization software}

Recording one second with the $120 \mathrm{~Hz}$ of acquisition frequency means storing nearly 0.5 billion of points (assuming 4 million points per frame). This way just one minute measurement can end up filling a 360 GB disk space. In order to handle such heavy 4D data, a specialized software called FRAMES was developed. It is used to analyze, process and visualize 3D and 4D measurements. An example view of 
the FRAMES GUI (Graphical User Interface) and opened 4D measurement is presented in figure 8.

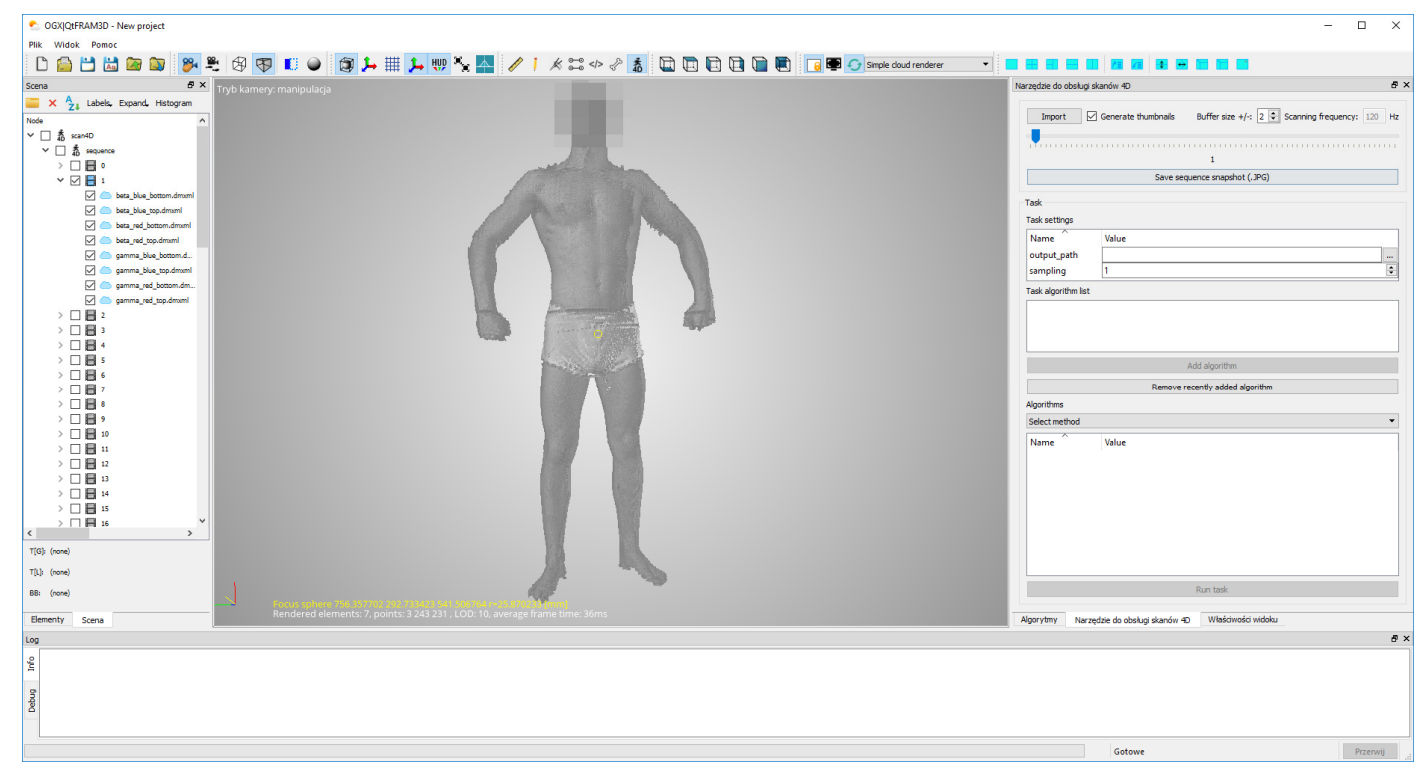

Fig. 8. The example view of the $4 D$ measurement in the FRAMES software.

Loading all of the 4D data at once is usually not possible due to a lack of available RAM (Random Access Memory). Therefore the data has to be loaded in chunks which are in fact separate frames. Only the currently viewed/analyzed frame and its buffer length predecessors and successors are stored in the memory. For an easier navigation over the whole sequence, reduced size thumbnails of each frame are generated during the measurement loading. While moving a timeline scrollbar, a live preview is rendered based on the thumbnails. This is fast enough and completely sufficient for the navigation purposes. When a tick of the timeline scrollbar is dropped, then the RAM is freed up and a chosen frame and its neighbors full data is loaded. Further visualization performance enhancements are possible thanks to a dynamic LoD (Level of Detail) mechanism. If rendering of the frame lasts too long, the FRAMES automatically decreases the quality of the displayed frame by halving a number of visible points. This is an iterative, gradual process that can be disabled. Figure 9 presents a 4D tool used for navigating over the sequence and running the processing algorithms.

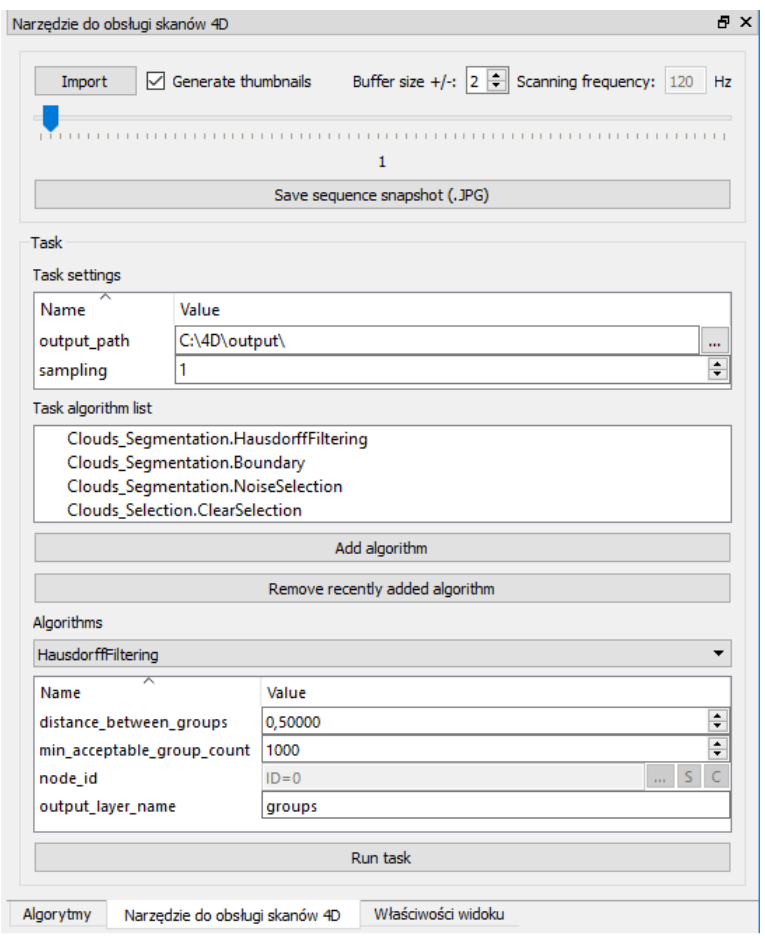

Fig. 9. The $4 D$ tool including navigation and algorithm sections. 
The FRAMES software enables both 3D and 4D advanced data processing. It is equipped with a set of useful algorithms implemented as a plugin system. This includes methods for a noise filtration, point cloud segmentation, manual and automatic point clouds adjustment, and many more. A list of the supported algorithms can be easily extended by using user-coded ones. It is possible thanks to a provided Software Development Kit (SDK) coming with a $\mathrm{C}_{++}$plugin wizard. The framework is also equipped with a scripting tool enabling fast prototyping of complex algorithm data flow paths. In order to shorten the processing time, multithreading is enabled whenever it is possible. The algorithms can be run individually on each frame as well as on the whole sequence. Of course, in addition to a standard $X$, $Y, Z$ 3D data, the methods can utilize the $4^{\text {th }}$ dimension (time) during the computation. An example view of the available FRAMES algorithms is shown in figure 10.

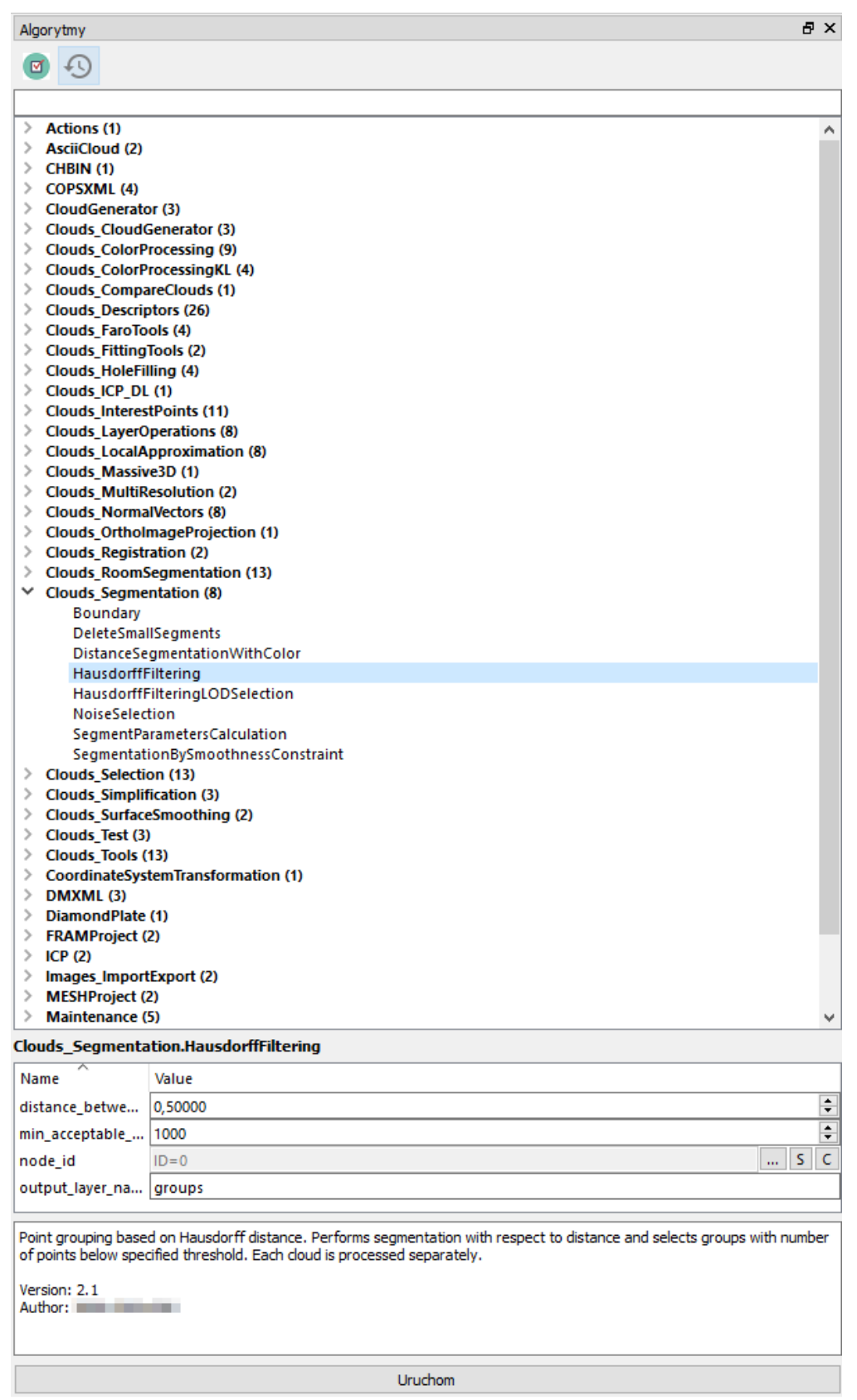

Fig. 10. The example view of the available FRAMES plugins. 


\section{Results}

The 4DBODY scanner can perform the 4D whole human body measurement with a frequency of up to $120 \mathrm{~Hz}$. The output point clouds consist of approximately four million points per frame and could be easily exported to commonly used formats, such as a triangle mesh. A spatial resolution is approximately $1.0 \mathrm{~mm}$ and a data inaccuracy is less than $0.5 \mathrm{~mm}$. The system is also equipped with the FRAMES application - a powerful 3D and 4D data processing software which makes the solution complete and ready to use product. Figure 11 presents three example frames from a captured 4D sequence demonstrating a quality of the system.
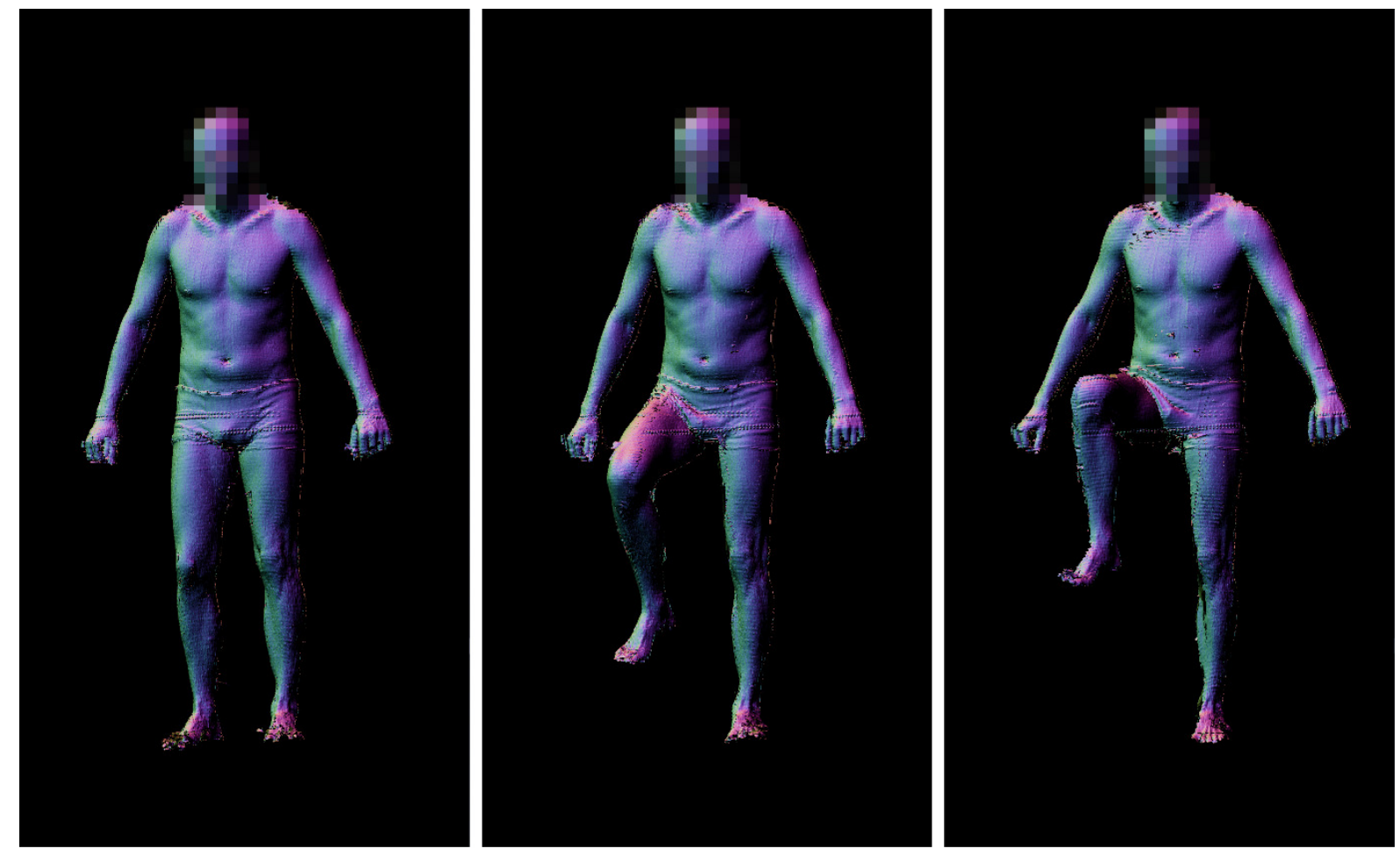

Fig. 11. Example measurement frames of a patient raising his leg.

\section{Conclusions}

The presented 4DBODY system is potentially usable in numerous medical applications, such as supporting medical rehabilitation process and diagnosis. Currently, tests in a real life environment are ongoing. Locomotor system rehabilitation data, such as after leg amputation, is now gathered using 4DBODY scanner. This kind of monitoring can potentially help to improve and speed up the rehabilitation process. However, the studies are not yet finished, hence not ready to be published. Moreover, new algorithms development is possible thanks to the added 4th dimension and the provided FRAMES analysis software. The proposed 4D whole body scanning solution can also be utilized in computer graphics, as a source of ready-to-use animated 3D models.

In the future, we plan to focus on the lowering of the system inaccuracy, optimization of our algorithms to reduce the processing time, and extension of the list of possible applications of the FRAMES software. We also consider increasing the number of the processing units and utilizing scattered processing in order to achieve a pseudo-real-time model reconstruction.

\section{Acknowledgment}

The work described in this article was part of the project PBS3/B9/43/2015, funded by the National Centre for Research and Development with public money for science and statutory work of IMiF Warsaw University of Technology. 


\section{References}

[1] P. Liberadzki, M. Adamczyk, M. Witkowski, and R. Sitnik, "Structured-light-based system for shape measurement of the human body in motion," Sensors, vol. 18, no. 9, 2018, http://doi.org/10.3390/s18092827.

[2] R. Zlot, M. Bosse, K. Greenop, Z. Jarzab, E. Juckes, and J. Roberts, "Efficiently capturing large, complex cultural heritage sites with a handheld mobile 3D laser mapping system," J. Cult. Herit., 2014, http://doi.org/10.1016/j.culher.2013.11.009.

[3] M. Adamczyk, M. Sieniło, R. Sitnik, and A. Woźniak, "Hierarchical, three-dimensional measurement system for crime scene scanning," J. Forensic Sci., 2017, http://doi.org/ 10.1111/1556-4029.13382.

[4] P. Treleaven and J. Wells, "3D body scanning and healthcare applications," Computer (Long. Beach. Calif)., 2007, http://doi.org/10.1109/MC.2007.225.

[5] J. Michoński, W. Glinkowski, M. Witkowski, and R. Sitnik, "Automatic recognition of surface landmarks of anatomical structures of back and posture," J. Biomed. Opt., 2012, http://doi.org/10.1117/1.JBO.17.5.056015.

[6] Ł. Markiewicz, M. Witkowski, R. Sitnik, and E. Mielicka, "3D anthropometric algorithms for the estimation of measurements required for specialized garment design," Expert Syst. Appl., vol. 85, 2017, http://doi.org/10.1016/j.eswa.2017.04.052.

[7] Q. Wei, J. Shan, H. Cheng, Z. Yu, B. Lijuan, and Z. Haimei, "A method of 3D human-motion capture and reconstruction based on depth information," in 2016 IEEE International Conference on Mechatronics and Automation, 2016, pp. 187-192, http://doi.org/10.1109/ICMA.2016.7558558.

[8] E. Ceseracciu, Z. Sawacha, and C. Cobelli, "Comparison of markerless and marker-based motion capture technologies through simultaneous data collection during gait: Proof of concept," PLoS One, 2014, http://doi.org/10.1371/journal.pone.0087640.

[9] C. Schaller, J. Penne, and J. Hornegger, "Time-of-flight sensor for respiratory motion gating," Med. Phys., vol. 35, no. 7, 2008, http://doi.org/10.1118/1.2938521.

[10] C. Bregler, A. Hertzmann, and H. Biermann, "Recovering non-rigid 3D shape from image streams," Proc. IEEE Comput. Soc. Conf. Comput. Vis. Pattern Recognit., 2000, http://doi.org/10.1109/CVPR.2000.854941.

[11]M. A.-B. Ebrahim, "3D laser scanners: history, applications, and future," 2011.

[12] J. Geng, "Structured-light 3D surface imaging: a tutorial," Adv. Opt. Photonics, vol. 3, no. 2, pp. 128-160, 2011, http://doi.org/10.1364/AOP.3.000128.

[13] M. Betsch, M. Wild, B. Johnstone, P. Jungbluth, M. Hakimi, B. Kühlmann, and W. Rapp, "Evaluation of a novel spine and surface topography system for dynamic spinal curvature analysis during gait," PLoS One, 2013, http://doi.org/10.1371/journal.pone.0070581.

[14]A. Gipsman, L. Rauschert, M. Daneshvar, and P. Knott, "Evaluating the reproducibility of motion analysis scanning of the spine during walking," Adv. Med., 2014, http://doi.org/10.1155/2014/721829.

[15] C. Zhang, S. Pujades, M. Black, and G. Pons-Moll, "Detailed, accurate, human shape estimation from clothed 3D scan sequences," in Proceedings - 30th IEEE Conference on Computer Vision and Pattern Recognition, CVPR 2017, 2017, http://doi.org/10.1109/CVPR.2017.582.

[16] G. Pons-Moll, J. Romero, N. Mahmood, and M. J. Black, "Dyna: a model of dynamic human shape in motion," ACM Trans. Graph., vol. 34, no. 4, 2015, http://doi.org/10.1145/2766993.

[17]I. Pachoulakis, "Augmented reality platforms for virtual fitting rooms," Int. J. Multimed. Its Appl., 2012, http://doi.org/10.5121/ijma.2012.4404.

[18]W. Glinkowski, J. Michonski, R. Sitnik, and M. Witkowski, "3D diagnostic system for anatomical structures detection based on a parameterized method of body surface analysis," Inf. Technol. Biomed. Adv. Intell. Soft Comput., vol. 69, 2010, http://doi.org/10.1007/978-3-642-13105-9 16.

[19]J. Lenar, M. Witkowski, V. Carbone, S. Kolk, M. Adamczyk, R. Sitnik, M. van der Krogt, and N. Verdonschot, "Lower body kinematics evaluation based on a multidirectional four-dimensional structured light measurement," J. Biomed. Opt., vol. 18, no. 5, 2013, http://doi.org/10.1117/1.JBO.18.5.056014. 
[20] "FLIR Machine Vision Cameras product specification web page." [Online]. Available: https://eu.ptgrey.com/grasshopper3-23-mp-mono-usb3-vision-sony-pregius-imx174. [Accessed: 31-Aug-2018]

[21] "Casio product specification web page." [Online]. Available: https://www.casio.com/products/projectors/slim-projectors/xi-a242. [Accessed: 31-Aug-2018].

[22] "Atmel AVR product specification web page." [Online]. Available: https://www.microchip.com/wwwproducts/en/ATxmega32E5. [Accessed: 31-Aug-2018].

[23] "Terasic FPGA product specification web page." [Online]. Available: https://www.terasic.com.tw/cgi-bin/page/archive.pl?Language=English\&No=1021. $\quad$ [Accessed: 31-Aug-2018].

[24] R. Sitnik, "Four-dimensional measurement by a single-frame structured light method," Appl. Opt., vol. 48, no. 18, pp. 3344-3354, 2009, http://doi.org/10.1364/AO.48.003344.

[25]N. Otsu, "A threshold selection method from gray-level histograms," IEEE Trans. Syst. Man. Cybern., vol. 9, no. 1, pp. 62-66, 1979, http://doi.org/10.1109/TSMC.1979.4310076.

[26] M. Takeda, "Spatial-carrier fringe-pattern analysis and its applications to precision interferometry and profilometry: An overview," Ind. Metrol., vol. 1, no. 2, pp. 79-99, 1990, http://doi.org/10.1016/0921-5956(90)80019-R.

[27]D. C. Ghiglia and M. D. Pritt, Two-dimensional phase unwrapping: theory, algorithms, and software, 1st ed. Hoboken, NJ, USA: Wiley-Interscience, 1998, http://doi.org/10.1177/004057368303900411.

[28] G. D. Bergland, "A guided tour of the fast Fourier Transform," IEEE Spectr., vol. 6, no. 7, pp. 41-52, 1969, http://doi.org/10.1109/MSPEC.1969.5213896.

[29]S. Rusinkiewicz and M. Levoy, "Efficient variants of the ICP algorithm," Proc. Int. Conf. 3-D Digit. Imaging Model. 3DIM, 2001, http://doi.org/10.1109/IM.2001.924423. 